\title{
An easy setup for double slide microarray hybridization
}

\author{
Abigail C. Ting ${ }^{1}$, Shern-Fwu Lee ${ }^{2}$, and Kai Wang ${ }^{1}$ \\ ${ }^{1}$ PhenoGenomics, Bothell, WA, USA and ${ }^{2}$ Taiwan Genome Sciences, Hsichih, Taiwan
}

BioTechniques 35:808-810(October 2003)

Since the inception of microarray technology, the amount of available gene expression information has skyrocketed. As a massive parallel platform for data gathering, microarrays make it possible to obtain data about global gene expression changes under varying physiological or pathological conditions (1-3). Gene probes labeled with fluorescent dye, along with microspotting devices, make microarray technology a sensitive and highthroughput tool for measuring expression level changes of tens of thousands of transcripts simultaneously (4).

A microarray experiment generally begins with gene targets (cDNA or oligonucleotides) spotted onto a processed microscope slide. A coverslip is then used to distribute the hybridization solution across the spotted area (array). Finally, the setup is placed in a humid hybridization chamber to maintain a stable microenvironment during hybridization. While microarray technology offers enormous increases in throughput, data quality still depends on various experimental conditions. These include the quality of printed DNA and processed microscope slides, environmental controls during printing, composition and temperature of hybridization solution, and the quality of the probes. All of these factors influence reproducibility, and often an experiment must be repeated numerous times to increase the data reliability (5-8).

Two-color fluorescent labeling has made microarray technology more powerful than the traditional singlelabeling hybridization procedure. However, a drawback of this fluorescence-based technology is that fluorescent probes are less sensitive than radioactively labeled ones (9). For this reason, microarray experiments generally require large amounts of RNA as the initial labeling material (sometimes up to $100 \mu \mathrm{g}$; see protocol in http:// cmgm.stanford.edu/pbrown/protocols/ 4_human_RNA.html). This can lead to difficulties when dealing with clinical samples, where the samples are often small and hard to obtain. It limits the potential for repeating experiments to increase the reliability of the data or probing different gene arrays to expand the gene coverage.

One way to alleviate these problems is to hybridize multiple slides using a single probe preparation. We are aware of one commercially developed double slide hybridization setup. In this arrangement, two arrayed slides are set face-to-face inside a chamber and secured by an attached comb-like clamp along each long edge of the unit. The teeth of the comb maintain space between the two slides. The hybridization solution can then be pipetted between the teeth and is drawn inside by capil-

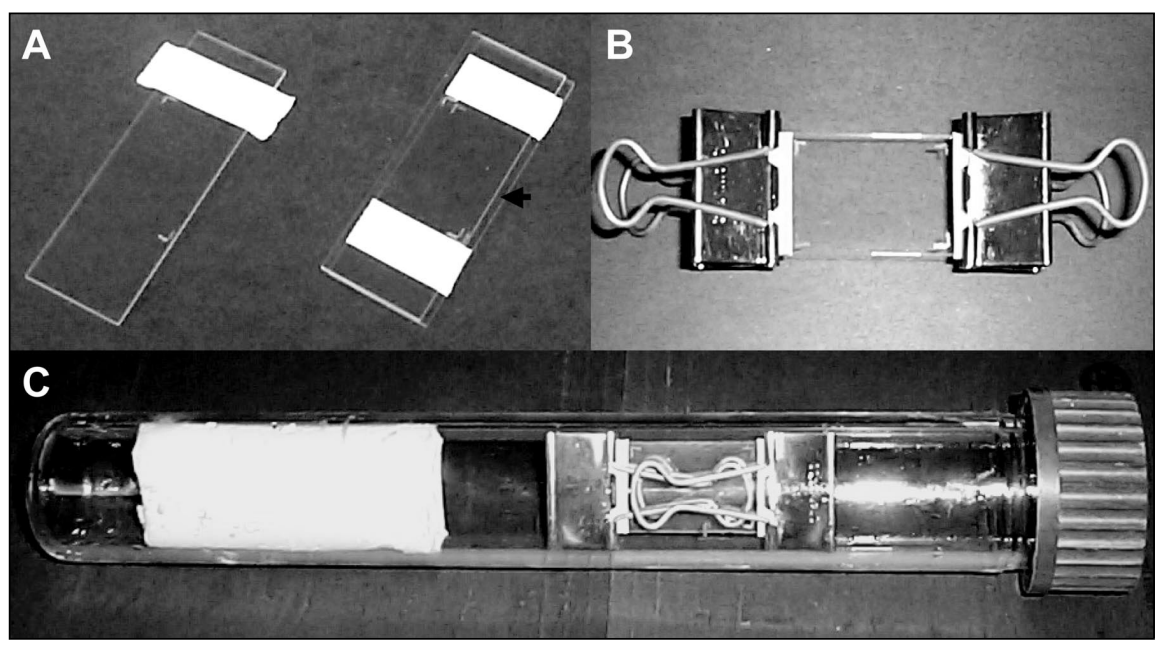

Figure 1. Assembly steps for a double slide hybridization unit. (A) Polytetra fluoroethylene tape is placed near the array printed on the first slide (two corners of the array are etched as shown). Second slide is placed on top of the tape with array side down. Tape on both ends of the arrays has been trimmed. The arrow indicates the offset area; the offset was made extra wide for demonstration purposes. (B) After trimming excess tape, both ends of the unit are clamped by stainless steel clips. (C) The assembled double slide unit can fit in a glass tube for a rotisserie hybridization oven. 
most of the drawbacks of the direct sandwich method by incorporating a thin spacer between the two slides. This spacer creates a fixed distance between the two slides and confines the solution within the arrayed area. This approach eliminates most of the unwanted effects that slide physical variations may have on array results.

In creating our double slide setup, the greatest challenge was finding a suitable material for the spacer. We required that the material be $(i)$ hydrophobic so as to repel hybridization buffer, (ii) inert to

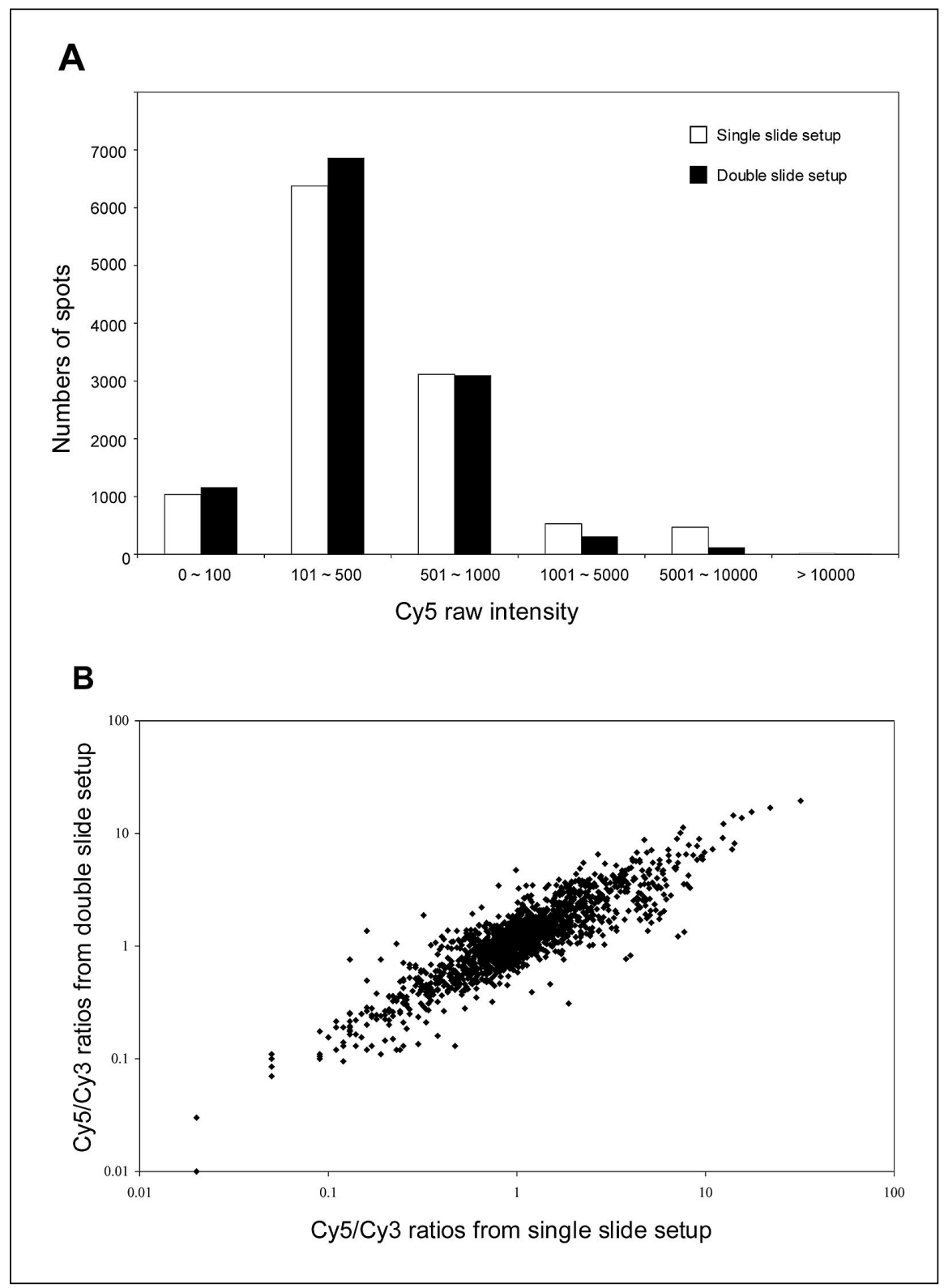

Figure 2. Comparison of hybridization results between traditional single slide and double slide setup. (A) Distribution of Cy5 raw intensity by number of gene spots. Double slide hybridization yields slightly lower intensities. (B) $\mathrm{Cy} 5 / \mathrm{Cy} 3$ ratios of validated gene spots from single slide vs. one slide of the double slide setup. The distribution of the ratios near the diagonal shows that the double slide setup yields comparable array data to the traditional single slide setup. The arrayed slides each contain 10,000 oligonucleotide spots printed in a 20- $\times 40-\mathrm{mm}$ area. Human liver and ovary total RNA (30 $\mu \mathrm{g}$ each) were reverse transcribed with amino-allyl dUTP, and then coupled with Cy5 and Cy3 fluorescent dyes, respectively. The labeled cDNA samples were resuspended in $80 \mu \mathrm{L}$ of hybridization solution and used to hybridize a single slide and a double slide setup simultaneously. The single slide setup was placed in a humid hybridization chamber (TeleChem International, Sunnyvale, CA, USA) and the double slide unit in a humid glass tube. Both were incubated in a rotisserie hybridization oven (Matrix Technologies) at $50^{\circ} \mathrm{C}$ overnight. 
most chemicals used in the experiments, (iii) as thin as possible to avoid potential probe dilution, (iv) versatile enough to fit arrays of different sizes, $(v)$ easy to handle, and (vi) readily available. We tested and discarded materials including thin plastic sequencing gel spacer, adhesive tape, silicon gel, and Parafilm $\mathrm{M}^{\circledR}$ (American National Can, Greenwich, CT, USA). These were rejected on the basis of undesirable properties such as potential for releasing unwanted chemicals, insufficient hydrophobicity, difficulty in handling, and heat intolerance.

In the end, we settled on polytetrafluoroethylene (PTFE; Teflon ${ }^{\circledR}$ ) thread tape. PTFE tape is a soft, stretchable material that is highly resistant to chemicals. It is also widely used as a sealant to prevent leakage of air, water, and chemicals. This tape is easy to handle and available in a variety of thicknesses. All these properties make PTFE tape a good candidate as spacer material in our double slide setup. It is important to note that the tape thickness dictates the volume of hybridization solution needed, so the thinnest tape available should be used. PTFE tape can be purchased at most hardware stores.

To assemble our double slide hybridization unit, the first slide is set with the arrayed side up. A strip of PTFE tape is placed at each end of the slide, slightly outside the arrayed area. The second slide is then placed on top of the tape with the arrayed side down and offset by at least $1 \mathrm{~mm}$ on the long side of the slide as shown in Figure 1A. The array on the second slide must also be placed within the taped area. The 1-mm slide offset is extremely helpful when loading the hybridization solution and separating the slides after hybridization. After the tape and slides are properly assembled, the tape should be trimmed to match the edge of the slide. Stainless steel clips (IDEA Scientific, Minneapolis, MN, USA) are placed on both ends of the slide to secure this double slide hybridization unit (Figure 1B). It is important that the clips clamp directly on the area above the two pieces of tape to ensure that pressure is applied evenly. The clips also serve as handles to allow moving the double slide unit without touching the slides. If desired, the unit may be warmed in a hybridization oven before applying the hybridization buffer.

The minimum volume of hybridization buffer required is calculated as the slide area between the two pieces of tape multiplied by the thickness of the tape (We routinely use $40 \mu \mathrm{L}$ with an area of $25 \times 40 \mathrm{~mm}$ between tape strips and a tape thickness of $0.04 \mathrm{~mm}$ ). After the probe has been prepared, it is applied directly onto the arrayed areas by slowly pipetting against the offset edge of the slides between the two tape strips. Surface tension retains the solution between the slides, preventing leakage.

For hybridization, we routinely place this double slide unit into a humid glass tube (Figure1C) and incubate the tube in a rotisserie hybridization oven (Matrix Technologies, Hudson, NH, USA) at $50^{\circ} \mathrm{C}$ overnight. We have not observed any drying of the hybridization solution within the arrayed area during our experiments over the past 8 months. To separate the double slide unit after hybridization, we remove the clips and insert a razor blade at an offset corner of the slides. Then we pry the slides apart and place them immediately into washing buffer for processing.

To demonstrate that our double slide setup would generate comparable array data to the traditional single slide method, we prepared a hybridization solution and used it to hybridize a single slide and a double slide setup simultaneously. Figure 2A shows the distribution of $\mathrm{Cy}^{\mathrm{TM}} 5$ raw intensity of each gene spot from the single slide and the average of the two slides from the double slide hybridization setup. The results indicate that the hybridization intensities from our double slide setup are slightly weaker than those from a traditional single slide setup. However, the lower intensities do not affect the $\mathrm{Cy} 5 / \mathrm{Cy} 3$ ratio as shown in Figure 2B. The Cy5/Cy3 ratios of the validated gene spots from the single slide were compared with the corresponding spots from the double slide unit. The coefficient of correlations between these two different approaches ranges from 0.89 to 0.92 . This suggests that our double slide setup yields array results of similar quality to those from traditional single slide hybridization.

This double slide setup also improves data reliability. For example, it helps eliminate false positive results.
If the gene expression pattern of a spot does not occur on both slides of a replicate pair, there is a good chance that the values of this gene are erroneous. Additionally, our approach increases the reliability of data by eliminating inconsistency caused by local variations generated during the array slide preparation (including the coating, spotting, and post-processing). We believe that using this simple double slide setup will facilitate array data analysis by reducing the number of false gene spots, as well as making the best use of a single probe preparation in routine array hybridization experiments.

\section{REFERENCES}

1.Butte, A. 2002. The use and analysis of microarray data. Nat. Rev. Drug Disc. 1:951-960.

2.Conway, T. and G.K. Schoolnik. 2003. Microarray expression profiling: capturing a genome-wide portrait of the transcriptome. Mol. Microbiol. 47:879-889.

3.Schofield, D. and T.J. Triche. 2002. cDNA microarray analysis of global gene expression in sarcomas. Curr. Opin. Oncol. 14:406-411.

4.Heller, M.J. 2002. DNA microarray technology: devices, systems, and applications. Annu. Rev. Biomed. Eng. 4:129-153.

5.Finkelstei D, R. Ewing, J. Gollub, F. Sterky, J.M. Cherry, and S. Somerville. 2002. Microarray data quality analysis: lessons from the AFGC project. Arabidopsis Functional Genomics Consortium. Plant Mol. Biol. 48: 119-131.

6.Gieser, P, G.C. Bloom, and E.N. Lazaridis. 2002. Introduction to microarray experimentation and analysis. Methods Mol. Biol. 184: 29-49.

7.Krajewski, P. and J. Bocianowski. 2002. Statistical methods for microarray assays. J. Appl. Genet. 43:269-278.

8.Quackenbush, J. 2002. Microarray data normalization and transformation. Nat. Genet. 32:496-501.

9.Murphy, K., T. Shimamura, and B.E. Bejcek. 2001. Use of fluorescently labeled DNA and a scanner for electrophoretic mobility shift assays. BioTechniques 30:504-511.

Received 7 April 2003; accepted 18 June 2003.

Address correspondence to Abigail C. Ting, PhenoGenomics, 22026 20th Ave. SE, Suite 101, Bothell, WA 98021, USA. e-mail: cnting@phenogenomics.com 